\title{
Acute generalized exanthematous pustulosis x Von Zumbusch's pustular psoriasis: A diagnostic challenge in a psoriatic patient*
}

\author{
Aline Soares de Sousa ${ }^{1}$ \\ Francine Papaiordanou ${ }^{2}$ \\ Antonio José Tebcherani ${ }^{1}$
}

\author{
Osório Alves Corrêa de Castro Lara ${ }^{1}$ \\ Gláucia Silva Simioni Marchioro ${ }^{1}$
}

DOI: http:/ /dx.doi.org/10.1590/abd1806-4841.20153256

\begin{abstract}
Severe cutaneous drug reactions include a wide spectrum of clinical manifestations ranging from mild morbilliform cutaneous rash, to severe forms of hypersensitivity. Special attention is given in this report to the acute generalized exanthematous pustulosis (AGEP), induced in $90 \%$ of cases by the use of systemic drugs, especially aminopenicillins and macrolides. The incidence of the disease is low, 1-5 cases per million patients / year. The main differential diagnosis is Von Zumbusch's Pustular Psoriasis. The prognosis is generally good and the disease self limited, after withdrawal of the triggering drug. In this report the authors describe a case of AGEP, triggered by ceftriaxone in a patient with psoriasis vulgaris.
\end{abstract}

Keywords: Drug hypersensitivity; Exanthema; Psoriasis

\section{INTRODUCTION}

Cutaneous drug reactions encompass a wide spectrum of clinical manifestations that range from mild morbilliform rash to severe forms of hypersensitivity ${ }^{1,2}$. Special attention is dedicated in this report to acute generalized exanthematous pustulosis (AGEP), which in over $90 \%$ of cases is induced by the use of systemic drugs, especially aminopenicillins and macrolides. ${ }^{1,-4}$

The term AGEP was first proposed by Beylot et al; later on Roujeau et al reported a series of 63 cases of the disease, in study published in 1991. 1,3,5 The first Brazilian case was described by Campbell and Furtado, who described how a patient developed AGEP following the use of ampicillin.

The incidence of the disease is 1 to 5 cases per million patients/year. The mean age of patients is 56 years and both genders are affected, with discreet female predominance. ${ }^{4}$ The etiopathogenesis is still not completely understood, although immune mechanisms mediated by $\mathrm{T}$ cells and cytokines have the greatest amount of scientific evidence up to the moment. $3,4,5,6$

The main differential diagnosis is Von Zumbusch's Pustular Psoriasis, formerly regarded as an AGEP variant, but clearly differentiated in more recent investigation (EuroScar study). ${ }^{6}$ Some criteria favor the pustular psoriasis diagnosis - history of psoriasis, prolonged fever, pustular rash of longer duration than in AGEP, absence of history of drug exposure and a differentiated histopathological picture. ${ }^{2,4,6}$ What makes diagnosis difficult is the fact that patients with psoriasis are more prone to develop AGEP than healthy patients, an affirmation based on statistical data, by means of a mechanism still unknown. $4,5,7$

The relationship established between the two nosological entities are the clinical similarities, but there is evidence that they are distinct diseases, as cited above. 
In this report the authors describe an AGEP case triggered by ceftriaxone in a patient with psoriasis vulgaris.

\section{CASE REPORT}

Male patient, 66 years old, from São Paulo, SP, with psoriasis vulgaris antecedent and previous treatment with acitretin. He was referred to the dermatology service due to the sudden onset of exanthema on the trunk and limbs, after use of ceftriaxone for treatment of erysipela. At first he was medicated with IV hydrocortisone, but even with the use of corticosteroids the lesions worsened and progressed with millimeter-sized pustules. The dermatological examination showed erythematous desquamative lesions on the trunk and limbs associated with disseminated pustules, more abundant in flexural areas (Figures 1 and 2). Laboratory tests revealed only leukocytosis, without deviations.

The patient was hospitalized with diagnostic hypotheses of AGEP $x$ generalized pustular psoriasis. Ceftriaxone was suspended and a cutaneous biopsy was performed, while introducing prednisone and antihistaminics orally.

The histopathological examination showed evidence of psoriasiform spongiotic dermatitis, with intense dermal edema and migration of neutrophils to the corneal layer. Correlating the findings with the clinical picture, the final diagnosis was AGEP (Figures 3 and 4 ).

The patient progressed with improved clinical picture and partial regression of the exanthema, as well as total regression of pustules in 5 days. He was discharged and remains under medical follow-up (Figure 5).

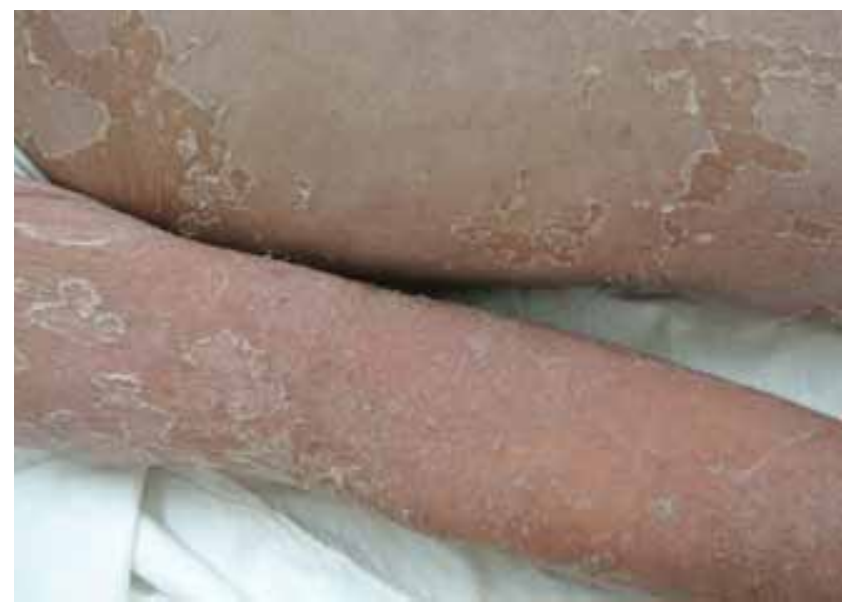

Figure 1: Pustules measuring approximately 0.1 to $0.2 \mathrm{~mm}$ and intense desquamation in erythematous skin

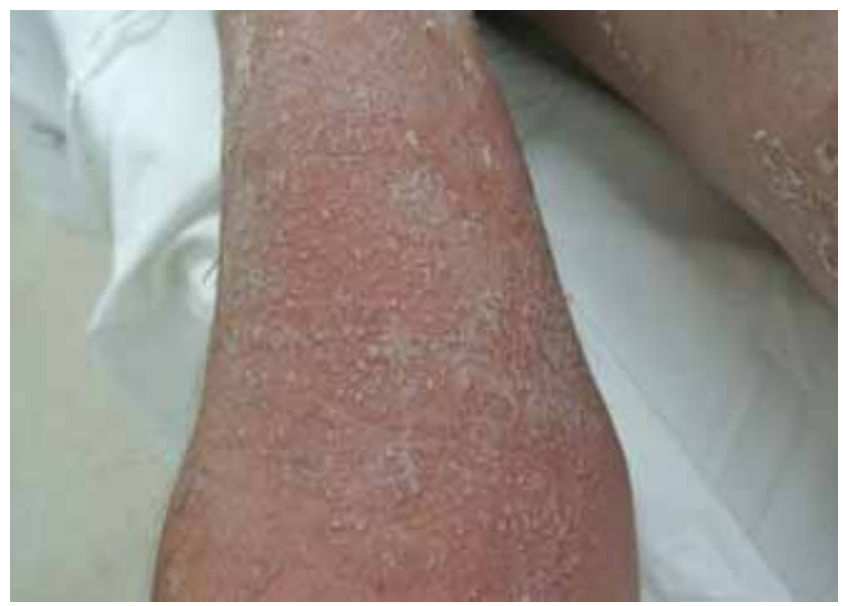

FiguRE 2: Detail of pustules concentrated in antecubital flexural region

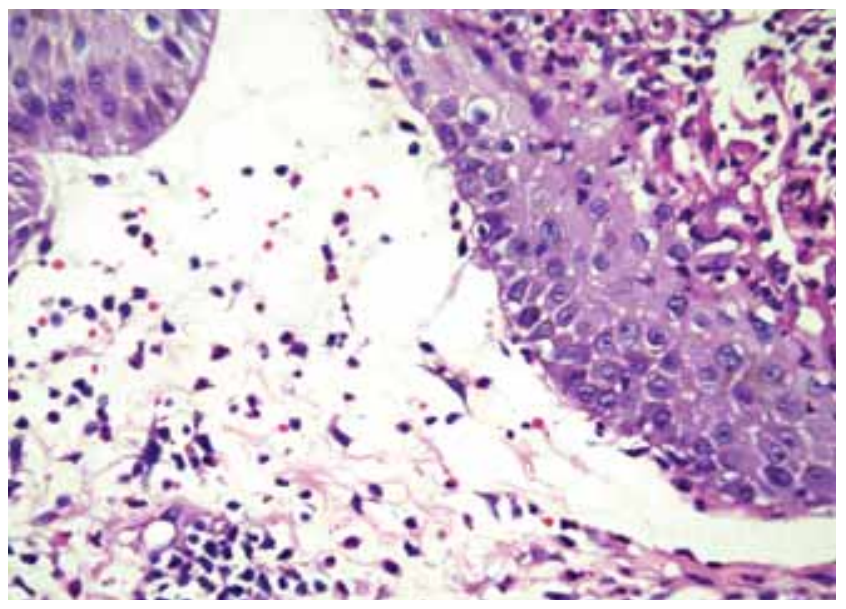

Figure 3: HE 100x. Epidermis with acanthosis, neutrophil migration and marked dermal edema

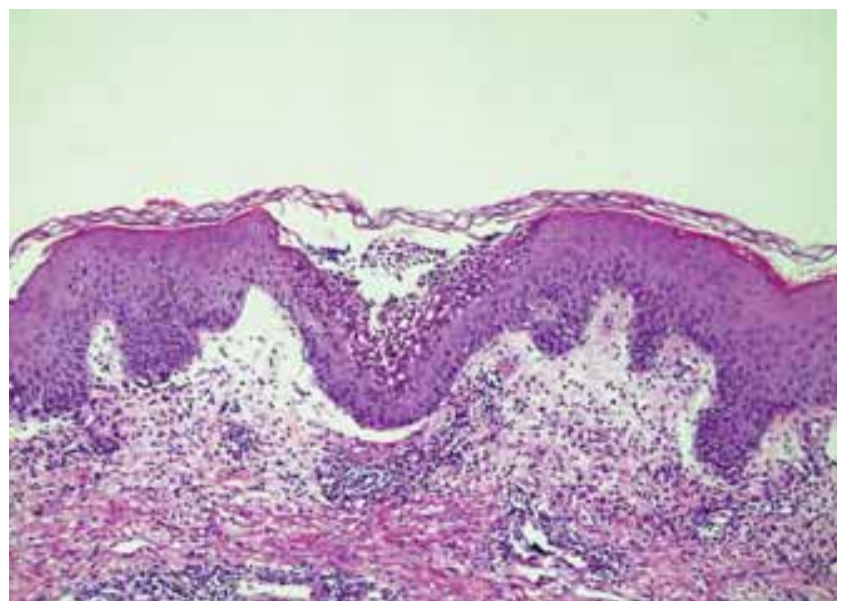

FIGURE 4: Detail of marked dermal edema and presence of eosinophils 


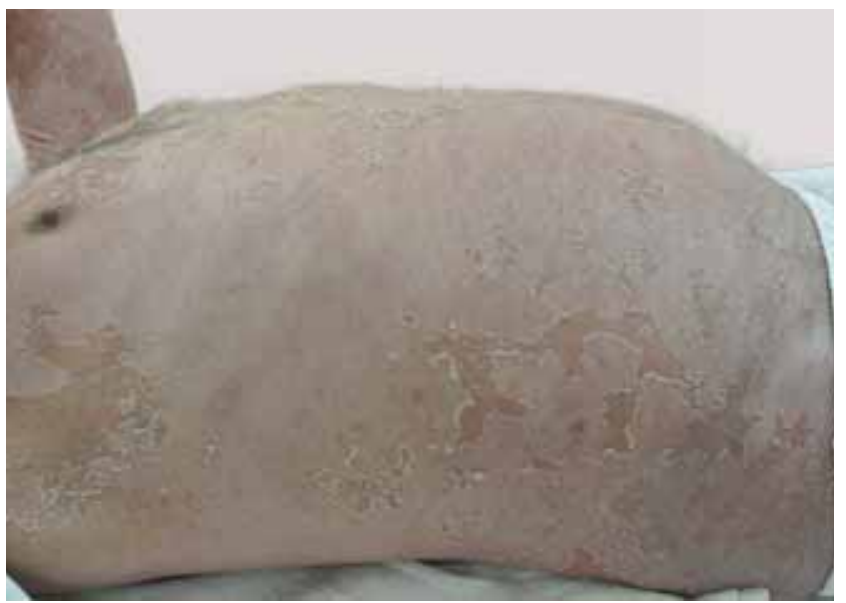

Figure 5: Trunk of patient with intense desquamation on erythematous base

\section{DISASSION}

The diagnosis of reaction to drugs is most times achieved after exclusion of other more probable diagnostic possibilities. In this case, some factors made immediate diagnosis difficult.

One of the main triggers of pustular psoriasis in patients with psoriasis vulgaris or even as the first manifestation of the disease is the untimely use of oral corticosteroid therapy. Our patient was treated with IV hydrocortisone for exanthema and progressed with pustulosis, but he had been medicated a few days before with antimicrobial from one of the main classes related to AGEP.

Some of the data suggest the AGEP diagnosis, but may not be considered as diagnostic criteria in view of their specificity in the differentiation with pustular psoriasis, still not tested in studies and not published in the literature. ${ }^{5}$

- Abrupt onset of pustular rash, hours or a few days after use of medication

- Hundreds of millimeter-sized non-follicular pustules, permeated by erythema and edema

- Leukocytosis with neutrophilia

- Pustule culture negative for bacteria

- Rapid resolution after drug suspension

The histopathologic standards that favor the AGEP diagnosis are: pustules

- Spongiform subcorneal or intraepidermal

- Eosinophils in the pustules or in the dermis

- Necrotic keratinocytes

- Neutrophilic infiltrate

- Absence of tortuous or dilated vessels
In pustular psoriasis, the rash is usually monomorphic, lasts for a longer period of time and is recurrent. It is less flexural than AGEP. Many times it is associated with arthritis and regresses in a period of 10 to 14 days. Histology shows epidermal hyperplasia, papillomatosis and acanthosis ${ }^{2,3}$. Other important differential diagnoses are: staphylococcal scalded skin syndrome, septicemia by staphylococcus aureus, bacterial folliculitis, subcorneal pustulosis, Stevens Johnson syndrome, toxic epidermal necrolisis and drug reaction with eosinophilia and systemic symptoms (DRESS). ${ }^{3,4,5}$

AGEP etiology is strongly associated with macrolides and beta-lactam based agents, but other medications and even viral infections may be related (quinolones, sulfonamides, terbinafine. antimalarials, calcium channel blockers, AINES, spider bites and infections by parvovirus B19, cytomegalovirus, coxsackie B4 and mycoplasma pneumoniae). ${ }^{1-8}$ It is important to remember that the main AGEP-triggering drugs are different from the main drugs implicated in the onset of other severe reactions, such as drug reaction with eosinophilia and systemic symptoms (DRESS) and toxic epidermal necrolisis (TEN), resulting mainly from the use of anticonvulsants, although antibiotics are also implicated.

The diagnosis is clinical, histopathologic, and in some cases the contact test, usually performed 4-6 weeks after the clinical picture is resolved, may help. A positive test may confirm the etiology, but a negative test does not exclude it.

The prognosis is good and the disease self limited, after withdrawal of the triggering drug, unless there is secondary infection of the lesions. A symptomatic treatment is indicated for relief of pruritus and cutaneous inflammation (medium potency topical corticosteroids $2 x$ / day for 1 week), besides antiseptic solutions in the pustular phase to prevent superinfection and emollients in the desquamative phase to reestablish the cutaneous barrier. Only rare severe cases require hospitalization for hydration support and electrolytic equilibrium.

This report stresses the need to include AGEP as possible diagnosis of patient with psoriasis presenting acute pustulosis, although generalized Von Zumbusch's Pustular Psoriasis is more prevalent in this group of patients. 


\section{REFERENCES}

1. Beltraminelli HS, Lerch M, Arnold A, Bircher AJ, Haeusermann P. Acute generalized exanthematous pustulosis induced by the antifungal terbinafine: case report and review of the literature. Br J Dermatol. 2005;152:780-3.

2. Criado PRi, Criado RFJ, Vasconcellos C, Ramos RO, Gonçalves AC. Severe cutaneous adverse drug reactions - relevant aspects to diagnosis and treatment Part II. An. Bras. Dermatol. 2004;79:587-601.

3. Belda Junior W, Ferolla AC. Acute generalized exanthematous pustulosis (AGEP). Case report. Rev Inst Med Trop Sao Paulo. 2005;47:171-6.

4. Sidoroff A. Acute generalized exanthematous pustulosis (AGEP). In: UpToDate. Mockenhaupt M, editors. Waltham, MA: UpToDate; 2013.

5. Buffon LP, Leal R. Acute Generalized Exanthematous Pustulosis (AGEP). An Bras Dermatol. 2002;77:223-8.

6. Sidoroff A, Dunant A, Viboud C, Halevy S, Bavinck JN, Naldi L, et al. Risk factors for acute generalized exanthematous pustulosis (AGEP) - results of a multinational case-control study (EuroSCAR). Br J Dermatol. 2007;157:989-96.

7. Razera F, OIm GS, Bonamigo RR. Dermatoses neutrofílicas: parte II. An Bras Dermatol. 2011;86:195-209.

8. Revuz J, Valeyrie-Allanore J. Urticarias, erythemas and purpuras. In: Bolognia JL, Jorizzo JL, Rapini RP, editors. Bolognia Textbook of Dermatology. 2nd ed. Spain: Mosby Elsevier publishing; 2008. p. 342-343.
M AILING ADDRESS:

Francine Papaiordanou

A v. Emílio Ribas, 1819

Gopouva

07050-000 Guarulhos, SP.

(11) 2408-2118

E-mail: francinepapaiordanou@gmail.com

How to cite this article: Sousa AS, Lara OACC, Papaiordanou F, Marchioro GSS, Tebcherani A. Acute generalized exanthematous pustulosis $\times$ Von Zumbusch's pustular psoriasis : A diagnostic challenge in a psoriatic patient. An Bras Dermatol. 2015;90(4):557-60. 